\title{
Physiological and Health-Related Adaptations to Low-Volume Interval Training: Influences of Nutrition and Sex
}

\author{
Martin J. Gibala $\cdot$ Jenna B. Gillen • \\ Michael E. Percival
}

(C) The Author(s) 2014. This article is published with open access at Springerlink.com

\begin{abstract}
Interval training refers to the basic concept of alternating periods of relatively intense exercise with periods of lower-intensity effort or complete rest for recovery. Low-volume interval training refers to sessions that involve a relatively small total amount of exercise (i.e. $\leq 10 \mathrm{~min}$ of intense exercise), compared with traditional moderate-intensity continuous training (MICT) protocols that are generally reflected in public health guidelines. In an effort to standardize terminology, a classification scheme was recently proposed in which the term 'highintensity interval training' (HIIT) be used to describe protocols in which the training stimulus is 'near maximal' or the target intensity is between 80 and $100 \%$ of maximal heart rate, and 'sprint interval training' (SIT) be used for protocols that involve 'all out' or 'supramaximal' efforts, in which target intensities correspond to workloads greater than what is required to elicit $100 \%$ of maximal oxygen uptake $\left(V \mathrm{O}_{2 \max }\right)$. Both low-volume SIT and HIIT constitute relatively time-efficient training strategies to rapidly enhance the capacity for aerobic energy metabolism and elicit physiological remodeling that resembles changes normally associated with high-volume MICT. Short-term SIT and HIIT protocols have also been shown to improve health-related indices, including cardiorespiratory fitness and markers of glycemic control in both healthy individuals and those at risk for, or afflicted by, cardiometabolic diseases. Recent evidence from a limited number of studies has highlighted potential sex-based differences in the adaptive response to SIT in particular. It has also been suggested that specific nutritional interventions, in
\end{abstract}

M. J. Gibala $(\bowtie) \cdot$ J. B. Gillen · M. E. Percival

Department of Kinesiology, McMaster University, Hamilton, ON L8S 4K1, Canada

e-mail: gibalam@mcmaster.ca particular those that can augment muscle buffering capacity, such as sodium bicarbonate, may enhance the adaptive response to low-volume interval training.

\section{Introduction}

Interval training has long been considered an essential component of programs designed to maximize performance in highly-trained athletes, which typically involve a relatively high volume of submaximal, moderate-intensity continuous training (MICT) [1-5]. While less well-appreciated, interval training per se is a potent stimulus to induce physiological remodeling that resembles-or indeed may be superior to-changes typically associated with traditional endurance training [6-9]. This brief commentary focuses on physiological and health-related adaptations to low-volume interval training, which is characterized by sessions that involve a relatively small total amount of exercise, compared with MICT protocols that are generally reflected in current public health guidelines [10-12]. The present work builds upon recent reviews by some of the same authors $[6,7,13]$ and also considers possible sexbased differences in the adaptive response to this type of training, in addition to the potential influence of nutritional manipulation on training outcomes. For a more comprehensive analyses of the physiological and health adaptations to interval training-and in particular comparisons between relatively high-volume interval training and traditional MICT, in which efforts are made to match energy expenditure-the reader is referred to recent reviews by others, including work that has focused on those at risk for, or afflicted by, cardiometabolic disorders such as cardiovascular disease and type 2 diabetes [8, 14-18]. Several other recent reviews have considered in detail various 
aspects of program design, with a particular focus on the application of interval training for athletic performance $[19,20]$.

\section{Characterizing the Training Stimulus: Standardizing Terminology}

Interval training refers to the basic concept of alternating periods of relatively intense exercise with periods of lowerintensity effort or complete rest for recovery. A wide range of terms have been used by different groups to describe various interval training protocols, which has led to a dizzying array of acronyms and general lack of standardization in the literature. Weston et al. [8] recently proposed a simple classification scheme for interval training based on exercise intensity as part of an effort to standardize terminology in future studies. The authors suggested that the term 'high intensity interval training' (HIIT) be used to describe protocols in which the training stimulus is "near maximal' or the target intensity is between 80 and $100 \%$ of maximal heart rate $\left(\mathrm{HR}_{\max }\right)$. In contrast, the authors advocated use of the term 'sprint interval training' (SIT) for protocols that involve 'all out' or 'supramaximal' efforts, in which target intensities correspond to workloads greater than what is required to elicit $100 \%$ of maximal oxygen uptake $\left(V \mathrm{O}_{2 \max }\right)$. Weston et al. [8] also suggested that the standardized term 'moderate-intensity continuous training' be used where appropriate in comparative studies. Other authors [21] have also recently considered various methodological approaches for the classification of interval training, including the use of turn-point or threshold models to prescribe intensity rather than percentages of $\mathrm{HR}_{\text {max }}$ or $V \mathrm{O}_{2 \max }$.

We applaud the efforts to try and standardize interval training terminology research in future studies. While cognizant of the potential value in other approaches [21, 22], especially for training prescription for athletes, we will employ the basic classification scheme proposed by Weston et al. [8] in the present review, given the widespread use of percentages of $\mathrm{HR}_{\max }$ and $V \mathrm{O}_{2 \max }$ to describe relative exercise intensity. Weston et al. [8] used the specific descriptors 'peak heart rate' and 'maximal oxygen uptake'; in the present review we will use ' $\mathrm{HR}_{\max }$ ' and ' $V \mathrm{O}_{2 \max }$ ' to describe relative intensities scaled to 'peak' and/or 'maximal' heart rate and oxygen uptake, respectively, for simplicity and consistency, and regardless of the specific term used in original studies that are cited here. There is no universal definition of what constitutes 'low volume' interval training, but in the present review we will consider protocols in which the total amount of intense exercise performed during a training session was $\leq 10$ min within a training session, i.e. the summed total duration of the hard efforts, excluding the recovery periods and any warm-up or cool-down. Based on this depiction and the classification scheme proposed by Weston et al. [8], an example of a low-volume HIIT protocol is ten 60-s cycling efforts at an intensity that elicits $\sim 85-90 \% \mathrm{HR}_{\max }$, interspersed by $60 \mathrm{~s}$ of recovery [23]. An example of low-volume SIT is the repeated Wingate Test model, which typically consists of four to six 30-s all-out efforts at mean power outputs corresponding to $\sim 250 \%$ of the absolute workload elicited at the end of an incremental $V \mathrm{O}_{2 \max }$, interspersed with a few minutes of recovery [24]. An overview of common protocols employed in interval training studies is depicted in Fig. 1.

\section{Physiological Adaptations to Low-Volume Interval Training}

It has been recognized for some time that relatively shortterm SIT and HIIT protocols can rapidly enhance the capacity for aerobic energy metabolism $[25,26]$ and elicit physiological remodeling that resembles changes induced by MICT. While relatively few direct comparisons have

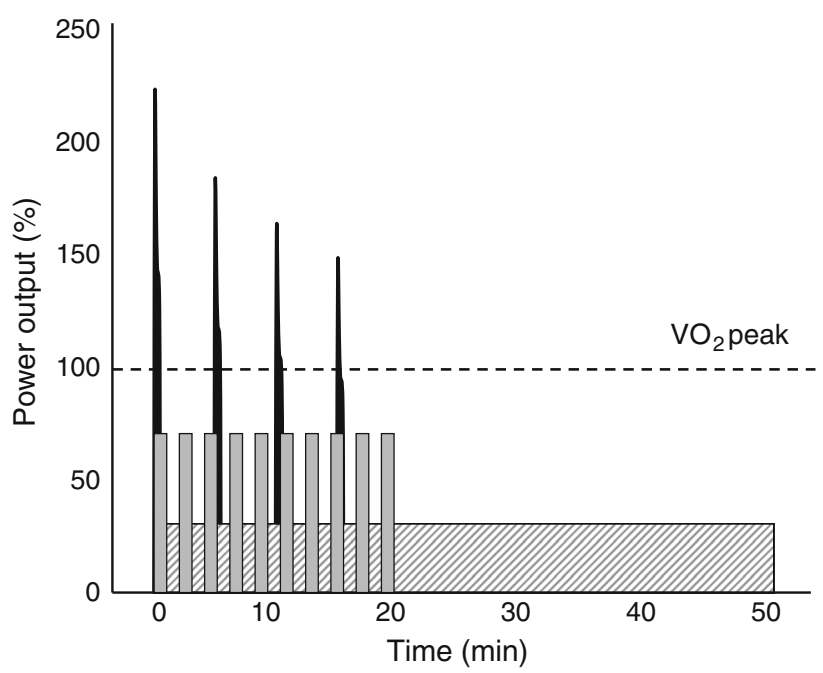

Fig. 1 Examples of protocols employed in interval training studies, expressed relative to PPO that is required to elicit $V \mathrm{O}_{2 \max }$ or $V \mathrm{O}_{2 \text { peak }}$. The figure shows typical MICT, e.g. $50 \mathrm{~min}$ at $\sim 35 \%$ of PPO, which elicits $\sim 70 \%$ of $\mathrm{HR}_{\max }$ (hatched box); low-volume HIIT, e.g. $10 \times 1 \mathrm{~min}$ at a constant workload corresponding to $\sim 75 \%$ of PPO, interspersed with $1 \mathrm{~min}$ of recovery, which elicits $\sim 85-90 \%$ of $\mathrm{HR}_{\max }$ during the intervals (grey bars); and low-volume SIT, e.g. $4 \times 30 \mathrm{~s}$ 'all out' effort at a variable power output corresponding to $\sim 175 \%$ of PPO (averaged over the course of the intervals), interspersed with 4 min of recovery, which elicits $\sim 90-95 \%$ of $\mathrm{HR}_{\max }$ during the intervals (black bars). Power output and heart rate estimates are derived from Little et al. [31] and Skelly et al. [60]. $P P O$, peak power output, $V O_{2 \max }$, maximal oxygen uptake, $\mathrm{VO}_{2}$ peak, peak $\mathrm{VO}_{2}, M I C T$, moderate-intensity continuous exercise, $H R_{\text {max }}$, maximum heart rate, HIIT, high-intensity interval training, SIT, sprint-interval training 
been made, in one of the first studies to compare skeletal muscle adaptations after low-volume interval versus traditional endurance training, Gibala et al. [27] reported increases in various markers of mitochondrial content after only six sessions of SIT or MICT over 2 weeks. Young active men performed either four to six Wingate Tests with 4 min of recovery in between, or 90-120 min of continuous cycling at approximately $65 \% V^{2} \mathrm{O}_{2 \max }$, per session. Total training time commitment was $\sim 2.5 \mathrm{~h}$ and $\sim 10.5 \mathrm{~h}$ for SIT and MICT, respectively, and total training volume based on mechanical work was $\sim 90 \%$ lower in the SIT group $(\sim 630$ vs. $\sim 6,500 \mathrm{~kJ})$. Skeletal muscle needle biopsy samples obtained before and after training revealed similar increases in the maximal activities of citrate synthase and cytochrome c oxidase (COX) and the protein content of COX subunits II and IV, with no difference between groups [27]. Similar adaptations have been reported in studies that examined skeletal muscle adaptations to 2-week HIIT protocols [23, 28]. Burgomaster et al. [29] compared two groups of previously sedentary individuals who performed either 6 weeks of Wingate-based SIT, 3 days/week similar to Gibala et al. [27], or MICT that involved 40-60 min of continuous cycling at $65 \%$ of $V \mathrm{O}_{2 \text { peak }}, 5$ days/week. Both protocols elicited similar increases in the maximal activities of mitochondrial markers, including citrate synthase, pyruvate dehydrogenase and 3-hydroxyacyl CoA dehydrogenase, and also reduced muscle glycogen and phosphocreatine utilization during submaximal, matched-work exercise following training in both groups.

The molecular mechanisms underlying skeletal muscle metabolic adaptations to low-volume interval training have been reviewed elsewhere [6], but in general many of the underlying signaling events appear to be at least qualitatively similar to processes proposed to regulate adaptations to MICT [31]. For example, similar acute activation of signaling pathways involved in mitochondrial biogenesis have been reported after a single session of SIT, HIIT, or MICT, including phosphorylation of 5'AMP (adenosine monophosphate)-activated protein kinase (AMPK), p38 mitogen-activated protein kinase (p38 MAPK), and p53 $[31,32]$. This suggests that at least some of the underlying mechanisms are qualitatively similar between the two training modalities, although it is also possible that interval training may stimulate pathways that initially differ from MICT but ultimately converge to elicit specific adaptive responses, e.g. mitochondrial biogenesis [3].

With respect to cardiovascular adaptations in healthy individuals, Rakobowchuk et al. [33] reported, in a companion paper that was based on the study by Burgomaster et al. [30], similar improvements in peripheral vascular structure and function, including popliteal artery distensibility and flow-mediated dilation, after 6 weeks of low- volume SIT and MICT. A subsequent study from another laboratory that employed the same experimental design [34] found that 6 weeks of SIT and MICT were equally effective in increasing skeletal muscle microvascular density and enzyme content, despite large differences in total training volume. Consistent with these findings, McKay et al. [35] reported that eight sessions of either SIT (eight to twelve 60-s intervals at $120 \% V^{2} \mathrm{O}_{2 \mathrm{max}}$, separated by $60 \mathrm{~s}$ of rest) or MICT (90-120 min at $65 \% V_{2 \max }$ ) improved muscle $\mathrm{O}_{2}$ utilization kinetics, suggestive of adaptations in local microvascular perfusion. Bailey et al. [36] also found that six sessions of Wingate-based SIT over 2 weeks accelerated muscle $\mathrm{O}_{2}$ utilization kinetics; however, no improvement was seen after MICT. With regards to central adaptations, MacPherson et al. [37] found that cardiac output, based on an acetylene non-rebreathing technique, was increased after 6 weeks of MICT but not SIT, despite similar improvements in $V \mathrm{O}_{2 \max }$. The authors suggested the early time course of cardiovascular adaptation to SIT and MICT may differ, with peripheral factors (i.e. enhanced $\mathrm{O}_{2}$ extraction) being more important for the former. In support of this interpretation, Jacobs et al. [38] showed that 2 weeks of SIT increased $V_{2} \mathrm{O}_{2 \max }$ and skeletal muscle respiratory capacity, but not cardiac output based on a nitrous oxide rebreathing method. In contrast, Esfandiari et al. [39] recently reported that Doppler-derived measures of end-diastolic volume, stroke volume, and cardiac output, as well as blood volume and $\mathrm{VO}_{2 \max }$, were increased to a similar extent after a 2 -week HIT protocol that was modeled after Little et al. [29], as well as a highvolume MICT protocol. While additional work is warranted to clarify the precise nature and time course of the mechanisms involved, a substantive body of evidence suggests that low-volume SIT and HIIT protocols constitute relatively time-efficient training strategies to induce an array of physiological adaptations that resemble changes normally associated with high-volume MICT, at least over the short-term, i.e. up to several weeks.

\section{Changes in Health-Related Indices After Low-Volume Interval Training}

Cardiorespiratory fitness has been documented to be a stronger predictor of risk for adverse health outcomes than traditional risk factors such as hypertension, smoking, obesity, and hyperlipidemia [40]. As little as six sessions of low-volume SIT over 2 weeks has been shown to improve cardiorespiratory fitness, as reflected by increased $V \mathrm{O}_{2 \max }$ during an incremental exercise test to exhaustion [36, 4143]. A recent systematic review and meta-analysis based on 16 studies summarized the improvements in cardiorespiratory fitness following SIT in young healthy individuals, 
and reported a moderate to large effect size in comparison to non-exercise control groups, and no difference when compared with MICT [44]. Interestingly, the effect of SIT on aerobic capacity was unaffected by initial fitness level (sedentary, recreational, trained), length of training intervention ( $<6$ weeks, $\geq 6$ weeks), or mode of training (cycling, running, rowing). The aggregate increase in $V \mathrm{O}_{2 \max }$ after SIT was $3.6 \mathrm{ml} / \mathrm{kg} / \mathrm{min}(8 \%)$, an improvement that would approximately translate into a 15 and $19 \%$ lower risk of all-cause and cardiovascular disease mortality, respectively [45]. Similar to the findings of Gist et al. [44], another meta-analysis of 19 studies by Sloth and colleagues [46] reported that $V \mathrm{O}_{2 \max }$ increased by a range of 4-13\% after 2-8 weeks of SIT in healthy sedentary or recreationally active adults. Data from individual studies, reminiscent of classic work by Tabata et al. [27], reveal that a surprisingly small total SIT dose can elicit relatively large changes in $V \mathrm{O}_{2 \max }$. For example, $\mathrm{Ma}$ et al. [47] recently showed that 16 sessions of a protocol that involved eight 20 -s cycling efforts at $170 \% V \mathrm{O}_{2 \max }$, interspersed with $10 \mathrm{~s}$ recovery, improved $V \mathrm{O}_{2 \max }$ in young men by $19 \%$ after 4 weeks. Similar improvements have also been reported using protocols involving $10 \mathrm{~s}$ [41] and $20 \mathrm{~s}$ allout cycling sprints [48]. There are less data on the effects of low-volume HIIT but recent studies showed improvements in $V \mathrm{O}_{2 \max }$ in overweight women after 6 weeks of training [49], and in patients with coronary artery disease after 12 weeks of training [50], with the latter change being comparable to a similar period of MICT that involved twice as much exercise. Relative intensity appears to be important when it comes to HIIT protocols, with a recent study showing that cardiovascular adaptation, as reflected by change in $V \mathrm{O}_{2 \max }$, was reduced when overweight/obese men trained using a $10 \times 60$-s protocol at an intensity equivalent to $70 \%$ of peak power elicited at $V \mathrm{O}_{2 \max }$ compared with $100 \%$ of peak power [51].

A few studies have reported improvements in glycemic control after short-term, low-volume interval training, in both healthy individuals and those at risk or afflicted by cardiometabolic diseases. Babraj et al. [52] were the first to report that six sessions of SIT over 2 weeks was a sufficient stimulus to improve insulin sensitivity in young active men, measured using oral glucose tolerance tests (OGTT). These findings were confirmed by Richards et al. [53], who showed that, using the hyperinsulinemic euglycemic clamp technique, insulin sensitivity was improved $72 \mathrm{~h}$ following a 2-week SIT intervention in healthy men and women. Whyte et al. [43] reported 2 weeks of SIT in overweight but otherwise healthy adults improved OGTT-derived estimates of insulin sensitivity when measured 24 but not $72 \mathrm{~h}$ after training, and Hood et al. [23] showed that six sessions of HIIT over 2 weeks improved insulin sensitivity based on fasting measures of glucose and insulin in previously sedentary individuals. Using continuous glucose monitoring (CGM), Little et al. [54] reported lower 24-h blood glucose concentration in patients with type 2 diabetes when measured $72 \mathrm{~h}$ following a 2-week HIIT protocol. All of these studies have been relatively short-term investigations on small numbers of subjects, without direct comparison with high-volume MICT protocols.

Recent evidence has also highlighted the potential for low-volume interval training to induce favorable changes in body composition. For example, 18 sessions of all-out running SIT over 6 weeks decreased whole body fat mass and increased whole body fat-free mass in recreationally active men [55] and women [56]. A cycling-based SIT protocol involving 60 repetitions of $8 \mathrm{~s}$ all-out sprints, interspersed with $12 \mathrm{~s}$ recovery, performed $3 \times /$ week for 15 weeks, was also shown to be more effective than an MICT protocol involving $40 \mathrm{~min}$ of cycling at $60 \%$ of $V \mathrm{O}_{2 \max }$ for decreasing whole body and abdominal fat mass in women [57]. Gillen et al. [49] also reported reductions in whole body and abdominal fat mass following 6 weeks of low-volume HIIT in overweight women. Boutcher [58] has discussed the various factors that could mediate changes in body composition after low-volume interval training, and some evidence has been presented in support of specific mechanisms, including increased post-exercise oxygen consumption or changes in appetite [59-61].

\section{Potential Sex-Based Differences in the Adaptive Response to Low-Volume Interval Training}

Recent evidence from a limited number of studies has highlighted potential sex-based differences in the adaptive response to SIT. Metcalfe et al. [48] utilized a protocol that involved two 20 -s all-out sprints within a 10-min training session that otherwise consisted of low-intensity cycling, including warm-up and cool-down. When previously sedentary but otherwise healthy men and women trained $3 \times 1$ week for 6 weeks, gains in aerobic capacity were similar, but insulin sensitivity measured using OGTTs was improved in men only. We also previously observed no change in OGTT-derived estimates of insulin sensitivity after a 6-week HIIT intervention in women [49], although this study did not involve a sex-based comparison. It has been suggested that high rates of glycogen breakdown and subsequent resynthesis following intense exercise may explain the rapid improvement in insulin sensitivity after SIT [48]. However, in comparison to men, women are reported to break down $42 \%$ less muscle glycogen in type 1 fibers during a single Wingate sprint [62], which is supported by a lower blood lactate accumulation following single [63, 64] and repeated 30-s sprints [64]. The reduced rate of glycogenolysis may be associated with lower basal 
activities of muscle phosphofructokinase [65] and lactate dehydrogenase reported in women [65, 66], or a lower catecholamine response to repeated sprints [63, 64]. Interestingly, women are suggested to have a greater predisposition for aerobic metabolism as estimates based on respiratory gas analyses suggest that the aerobic contribution to a 30 -s sprint is $25 \%$ higher in women compared with men [67]. This difference could also explain in part the relative glycogen sparing that has been observed in women [62], with potential effects of associated metabolic byproducts on downstream signaling events that regulate muscle adaptations. Divergent adaptations in skeletal muscle remodeling were reported following a 3-week SIT intervention in active young men and women. Oral administration of deuterium oxide revealed higher rates of muscle protein synthesis in men over the course of training in both the mixed and cytosolic fractions [68]. However, it is important to note that other studies involving mixed cohorts of men and women have not described sex-based differences in the adaptive response to interval training [23, 29, 53], although these studies were not specifically designed to address this issue. Clearly, additional wellcontrolled studies are warranted to determine whether women might in fact 'respond less' to low-volume SIT, using best practice designs that control for various factors, such as menstrual cycle phase and relative fitness, that can increase variance and lead to false conclusions regarding potential sex differences [69].

\section{Can Nutrition Alter the Adaptive Response to Low-Volume Interval Training?}

Based on our understanding of the molecular biology underlying muscle adaptation to exercise training [31], and the potential for nutrition to modulate training adaptation [70], interventions in theory could augment the adaptive response to interval training by: (1) improving energy metabolism during exercise, which could facilitate greater total work and an enhanced chronic training stimulus; or (2) promoting some aspect of the adaptive response during recovery, which could lead to enhanced physiological adaptations over time [71]. A large number of potential candidates, including, for example, caffeine and creatine, have been identified and discussed in detail elsewhere [7274]. For the purposes of this review, only a few specific nutritional practices or supplements will be considered here.

Arguably the best evidence to date regarding the potential for nutritional manipulation to enhance physiological adaptation to interval training is research on carbohydrate (CHO) restriction protocols [75]. As originally proposed by Hansen et al. [76], the basic concept is that training in a CHO-restricted state and/or with reduced glycogen availability could serve to augment the acute molecular signaling response to exercise [76]. A typical research design involves a comparison of two groups who either train once daily, or twice a day, every other day, for up to several weeks, with the manipulation resulting in the latter performing approximately half of all training sessions in a 'reduced' state [77-79]. These studies have generally failed to show any beneficial effect on performance, at least using whole-body exercise protocols that resemble normal athletic competition, and indeed perception of effort may be increased. However, from a basic science standpoint, studies have shown that $\mathrm{CHO}$-restricted training can enhance mitochondrial adaptation, even in highly trained individuals. This could result from the transient yet repeated enhancement of acute signaling proteins that regulate mitochondrial biogenesis. For example, several studies have shown that the acute activation of AMPK [80], p38MAPK [81], and p53 [82] is amplified when interval training is commenced with low glycogen per se or reduced CHO availability. Specifically in regards to AMPK regulation, the enhanced signaling may be mediated through a greater liberation of the regulatory beta subunit, which is sequestered under normal glycogen conditions [83-85].

Interventions that alter buffering capacity may also be of particular relevance to interval training. Repeated sprints have the potential to depress intramuscular $\mathrm{pH}$ to $6.5-6.8$ [86-89], which may reduce glycolytic flux [87, 88] and alter calcium sensitivity/handling $[90,91]$. However, the extent to which $\mathrm{pH}$ may play a role in fatigue has been questioned [89, 92, 93]. Nonetheless, elevated buffering capacity has been associated with enhanced sprint ability [94] and short duration endurance cycling performance [95]. Hence, research has focused on supplementing the natural buffering system in an attempt to augment physiological adaptations and performance. One method of manipulating the extracellular buffering capacity is through ingestion of sodium bicarbonate $\left(\mathrm{NaHCO}_{3}\right)$, as the associated elevation of blood bicarbonate, $\mathrm{pH}$ and base excess has been proposed to elevate $\mathrm{H}^{+}$efflux out of contracting muscles $[96,97]$. Another hypothesis is that alkalosis may help maintain muscle membrane excitability through improved strong ion regulation [98-100]. Regardless of the potential underlying mechanism, there is good evidence to support the practice of acute $\mathrm{NaHCO}_{3}$ ingestion, with a recent meta-analysis finding that a dose of $0.3-0.5 \mathrm{~g} / \mathrm{kg}$ body mass improved mean power output by $1.7 \%$ $( \pm 2.0 \%)$ during short high-intensity exercise [101]. However, it must be emphasized that not all studies have found performance improvements after $\mathrm{NaHCO}_{3}$ ingestion, and individual responses are variable [102].

With respect to the evidence that repeatedly induced metabolic alkalosis can augment adaptations over the 
Fig. 2 Hypothesized acute signaling mechanism underpinning enhanced training adaptations in response to chronic $\mathrm{NaHCO}_{3}$ supplementation. a AMPK acts as an energy sensor that is activated by various signals generated during muscle contraction (e.g. increased AMP), which subsequently activates PGC- $1 \alpha$, leading to increased transcription of various genes involved in mitochondrial biogenesis. b Supplementation with $\mathrm{NaHCO}_{3}$ may alter muscle metabolism, resulting in greater increases in AMP, which could enhance AMPK activation through interaction with the $\gamma$ subunit. Glycogen utilization during exercise is also increased after $\mathrm{NaHCO}_{3}$ supplementation, and the AMPK $\beta$-subunit that is sequestered by glycogen may be liberated to a greater extent. Greater AMPK activation could result in greater downstream signaling, including activation of PGC- $1 \alpha$ and increased gene expression. Red arrows depict potential mechanisms that are supported by experimental data, whereas question marks indicate areas that remain to be directly investigated. $\mathrm{NaHCO}_{3}$ sodium bicarbonate, $A M P K$ adenosine monophosphate kinase, $P G C-1 \alpha$ peroxisome proliferator-

activated receptor $\gamma$ co-activator
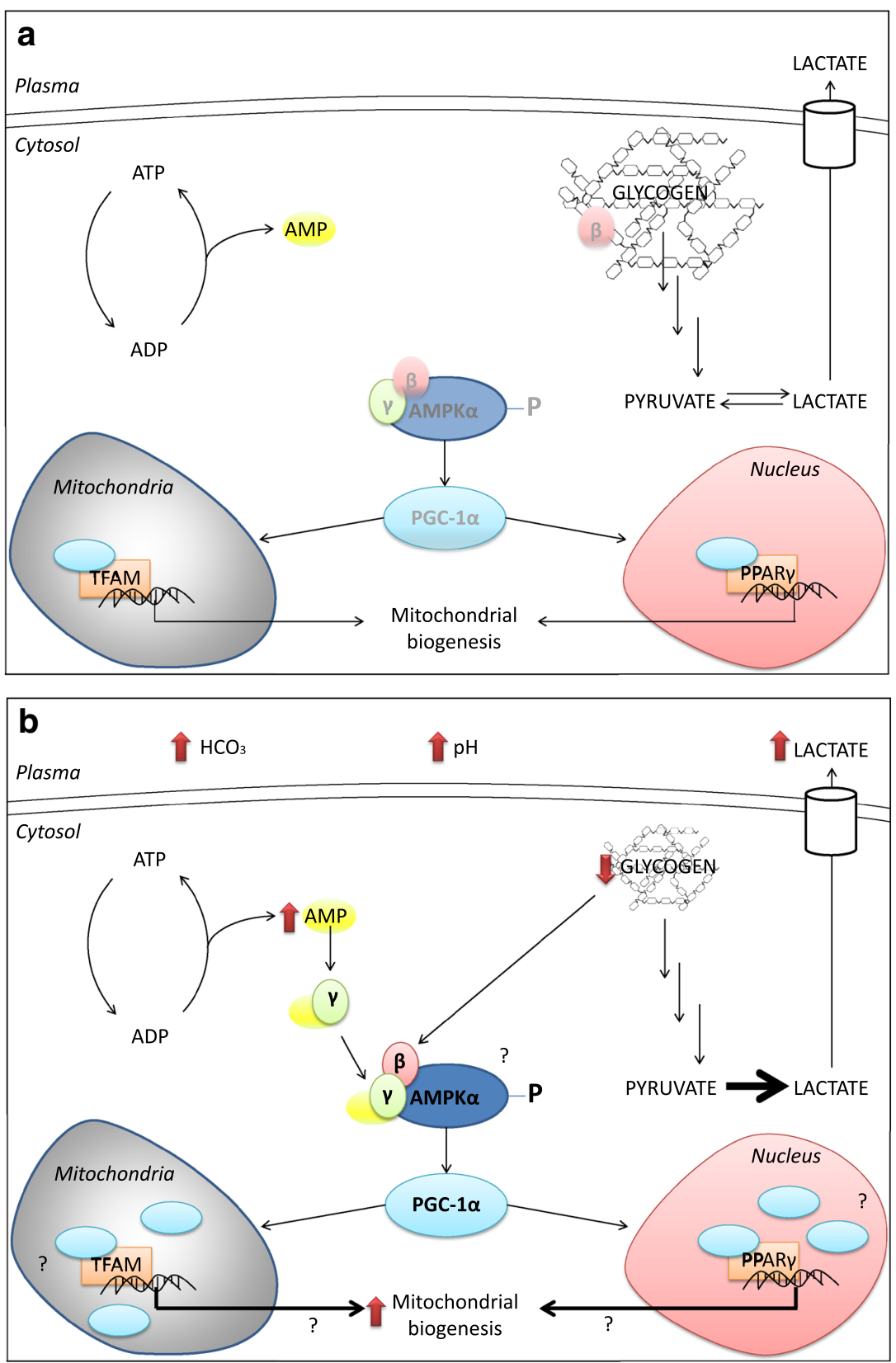

course of chronic training [103-105], Edge et al. [103] reported that recreationally active women who consumed $\mathrm{NaHCO}_{3}$ for 8 weeks in conjunction with interval training experienced lower $\mathrm{H}^{+}$accumulation during each session, and this was associated with a training-induced improvement in endurance capacity and lactate threshold. To garner insight into the potential mechanistic basis for the observed performance enhancement, Bishop and colleagues [105] had rats perform interval training five times/ week for 5 weeks while supplemented with $\mathrm{NaHCO}_{3}$ or a placebo. Both groups increased endurance capacity, as evidenced by significant increases in running time to exhaustion in comparison to unexercised control rats. However, the rats supplemented with $\mathrm{NaHCO}_{3}$ showed a superior performance improvement, attributed to greater improvements in both mitochondrial mass and respiration [104]. It is important to note that within both studies, groups were matched for total work performed during each training session, and thus the enhanced muscle adaptations and performance cannot be attributed to differences in total training volume.

Several studies have investigated the effects of $\mathrm{NaHCO}_{3}$ on muscle metabolism during acute exercise and these provide additional insight into potential cellular mechanisms involved [96, 97, 106-108]. A relatively common finding is that $\mathrm{NaHCO}_{3}$ supplementation increases the rate of muscle glycogen degradation [96, 97], and this is associated with increased content of lactate in muscle and/ 
or blood [96, 97, 106, 107]. Hollidge-Horvat et al. [96] also reported that exercise following $\mathrm{NaHCO}_{3}$ supplementation resulted in elevated muscle content of adenosine diphosphate, adenosine monophosphate, and inorganic phosphate, while phosphocreatine content was reduced in comparison to placebo. The authors proposed that altered cellular energetics were due to higher glycogen utilization, facilitated by a lack of inhibition on glycolytic flux, which conversely reduced free fatty acid (FFA) metabolism [96]. In support of this interpretation, the authors also found a marked decline in free carnitine as well as muscle $\mathrm{pH}$ following supplementation. Both mechanisms have been implicated in the inhibition of carnitine palmitoyltransferase 1 (CPT-1), the rate-limiting enzyme of long-chain fatty acid transport into mitochondria for beta oxidation [109112]. Hollidge-Horvat and colleagues further suggested that decreased FFA utilization would cause a decline in the ratio of reduced/oxidized nicotinamide adenine dinucletides in the mitochondria, requiring the observed alterations in cellular energetics to drive oxidative phosphorylation [96]. The hypothesized acute signaling mechanism underpinning enhanced training adaptations in response to chronic $\mathrm{NaHCO}_{3}$ supplementation is depicted in Fig. 2.

It has also been speculated that nutritional manipulation of intramuscular buffering capacity could augment adaptations to interval training. The dipeptide carnosine is an important intramuscular buffer, and the intramuscular content can be increased through chronic oral supplementation with the rate-limiting amino acid precursor, $\beta$-alanine [113]. A recent meta-analysis suggests that $\beta$-alanine has ergogenic effects on exercise lasting 1-4 min with an improvement of $2.85 \%$ in performance measures [114]. While the majority of studies have focused on performance, recent evidence suggests metabolism within the contracting muscle may be altered by $\beta$-alanine supplementation. For example Gross et al. [115] found that in response to a single, high-intensity, fixed-power, fixedduration test, supplementation with $\beta$-alanine appeared to reduce the oxygen deficit and accumulation of both blood and muscle lactate. The authors suggested that an enhancement in oxidative phosphorylation coinciding with a decrease in substrate level phosphorylation was evident [115]. Interestingly, these results appear to contrast those found when manipulating the extracellular buffer with $\mathrm{NaHCO}_{3}$ supplementation, as discussed above. Aside from this initial work by Gross and colleagues, very little is known regarding other acute metabolic effects that may influence muscle signaling [115]. Recent investigations using relatively short training blocks lasting up to several weeks suggest that chronic $\beta$-alanine supplementation does not further enhance the adaptive response [115-117].

In summary, while some evidence suggests that specific nutritional interventions may alter the adaptive response to interval training, future work should focus on elucidating the underlying mechanisms as well as understanding how these may translate to increased performance during athletic competition.

\section{Conclusions}

The majority of low-volume interval training studies have utilized relatively short intervention periods (i.e. lasting up to several weeks) and future work involving long-term (i.e. months to years) interventions is needed to advance our basic understanding of how manipulating the exercise stimulus translates into physiological remodeling. From an applied perspective, there is value in trying to establish the minimum 'dose' of HIIT or SIT needed to stimulate meaningful improvements in clinical markers that are associated with disease risk. This is particularly germane given that 'lack of time' remains the most commonly cited barrier to regular exercise participation [118], and considering evidence that suggests that low-volume interval training is perceived to be more enjoyable than MICT [119]. There is also evidence that nutritional interventions can influence both acute and chronic adaptations to interval training [74], findings that may have relevance beyond athletic performance and competitive sport. As suggested by van Loon and Tipton [120], greater adaptation efficiency has clinical relevance, especially for individuals with severe exercise intolerance.

Acknowledgments This article was published in a supplement supported by the Gatorade Sports Science Institute (GSSI). The supplement was guest edited by Lawrence L. Spriet who attended a meeting of the GSSI Expert Panel in February 2013 and received honoraria from the GSSI, a division of PepsiCo, Inc., for his meeting participation and the writing of his manuscript. He has not received any honoraria for guest editing the supplement. Lawrence L. Spriet, selected peer reviewers for each paper and managed the process. Martin J. Gibala, PhD, attended a meeting of the GSSI Expert Panel in February 2013 and received honoraria from the GSSI, a division of PepsiCo, Inc., for his meeting participation and the writing of this manuscript. The views expressed in this manuscript are those of the author and do not necessarily reflect the position or policy of PepsiCo, Inc.

Open Access This article is distributed under the terms of the Creative Commons Attribution License which permits any use, distribution, and reproduction in any medium, provided the original author(s) and the source are credited.

\section{References}

1. Iaia FM, Bangsbo J. Speed endurance training is a powerful stimulus for physiological adaptations and performance improvements of athletes. Scand J Med Sci Sports. 2010;20(Suppl 2):11-23. 
2. Kubukeli ZN, Noakes TD, Dennis SC. Training techniques to improve endurance exercise performances. Sports Med. 2002;32:489-509.

3. Laursen PB. Training for intense exercise performance: highintensity or high-volume training? Scand J Med Sci Sports. 2010;20(Suppl 2):1-10.

4. Laursen PB, Jenkins DG. The scientific basis for high-intensity interval training: optimising training programmes and maximising performance in highly trained endurance athletes. Sports Med. 2002;32:53-73.

5. Ross A, Leveritt M. Long-term metabolic and skeletal muscle adaptations to short-sprint training: implications for sprint training and tapering. Sports Med. 2001;31:1063-82.

6. Gibala MJ, Little JP, MacDonald MJ, et al. Physiological adaptations to low-volume, high-intensity interval training in health and disease. J Physiol. 2012;590:1077-84.

7. Gibala MJ, Jones AM. Physiological and performance adaptations to high-intensity interval training. Nestle Nutr Inst Workshop Ser. 2013;76:51-60.

8. Weston KS, Wisløff U, Coombes JS. High-intensity interval training in patients with lifestyle-induced cardiometabolic disease: a systematic review and meta-analysis. Br J Sports Med. 2014;48:1227-34.

9. Wisløff U, Ellingsen Ø, Kemi OJ. High-intensity interval training to maximize cardiac benefits of exercise training? Exerc Sport Sci Rev. 2009;37:139-46.

10. Garber CE, Blissmer B, Deschenes MR, et al. American College of Sports Medicine position stand. Quantity and quality of exercise for developing and maintaining cardiorespiratory, musculoskeletal, and neuromotor fitness in apparently healthy adults: guidance for prescribing exercise. Med Sci Sports Exerc. 2011;43:1334-59.

11. O'Donovan G, Blazevich AJ, Boreham C, et al. The ABC of Physical Activity for Health: a consensus statement from the British Association of Sport and Exercise Sciences. J Sports Sci. 2010;28:573-91.

12. Tremblay MS, Warburton DE, Janssen I, et al. New Canadian physical activity guidelines. Appl Physiol Nutr Metab. 2011;26:36-58.

13. Gillen JB, Gibala MJ. Is high-intensity interval training a timeefficient exercise strategy to improve health and fitness? Appl Physiol Nutr Metab. 2014;39:409-12.

14. Guiraud T, Nigam A, Gremeaux V, et al. High-intensity interval training in cardiac rehabilitation. Sports Med. 2012;42:587-605.

15. Haykowsky MJ, Timmons MP, Kruger C, et al. Meta-analysis of aerobic interval training on exercise capacity and systolic function in patients with heart failure and reduced ejection fractions. Am J Cardiol. 2013;111:1466-9.

16. Hwang $\mathrm{CL}, \mathrm{Wu} \mathrm{YT}$, Chou $\mathrm{CH}$. Effect of aerobic interval training on exercise capacity and metabolic risk factors in people with cardiometabolic disorders: a meta-analysis. J Cardiopulm Rehabil Prev. 2011;31:378-85.

17. Kessler HS, Sisson SB, Short KR. The potential for highintensity interval training to reduce cardiometabolic disease risk. Sports Med. 2012;42:489-509.

18. Meyer P, Gayda M, Juneau M, et al. High-intensity aerobic interval exercise in chronic heart failure. Curr Heart Fail Rep. 2013;10:130-8.

19. Buchheit M, Laursen PB. High-intensity interval training, solutions to the programming puzzle: part I: cardiopulmonary emphasis. Sports Med. 2013;43:313-38.

20. Buchheit M, Laursen PB. High-intensity interval training, solutions to the programming puzzle: part II: anaerobic energy, neuromuscular load and practical applications. Sports Med. 2013;43:927-54.
21. Tschakert G, Hofmann P. High-intensity intermittent exercise: methodological and physiological aspects. Int J Sports Physiol Perform. 2013;8:600-10.

22. Mann T, Lamberts RP, Lambert MI. Methods of prescribing relative exercise intensity: physiological and practical considerations. Sports Med. 2013;43:613-25.

23. Hood MS, Little JP, Tarnopolsky MA, et al. Low-volume interval training improves muscle oxidative capacity in sedentary adults. Med Sci Sports Exerc. 2011;43:1849-56.

24. Burgomaster KA, Hughes SC, Heigenhauser GJF, et al. Six sessions of sprint interval training increases muscle oxidative potential and cycle endurance capacity. J Appl Physiol. 2005;98:1895-900.

25. MacDougall JD, Hicks AL, MacDonald JR, et al. Muscle performance and enzymatic adaptations to sprint interval training. J Appl Physiol. 1998;84:2138-42.

26. Tabata I, Nishimura K, Kouzaki M, et al. Effects of moderateintensity endurance and high-intensity intermittent training on anaerobic capacity and $V \mathrm{O}_{2 \max }$. Med Sci Sports Exerc. 1996;28:1327-30.

27. Gibala MJ, Little JP, van Essen M, et al. Short-term sprint interval versus traditional endurance training: similar initial adaptations in human skeletal muscle and exercise performance. J Physiol. 2006;575:901-11.

28. Little JP, Safdar AS, Wilkin GP, et al. A practical model of lowvolume high-intensity interval training induces mitochondrial biogenesis in human skeletal muscle: potential mechanisms. J Physiol. 2010;586:1011-22.

29. Burgomaster KA, Howarth KR, Phillips SM, et al. Similar metabolic adaptations during exercise after low volume sprint interval and traditional endurance training in humans. J Physiol. 2008;586:151-60.

30. Egan B, Zierath JR. Exercise metabolism and the molecular regulation of skeletal muscle adaptation. Cell Metab. 2013;17:162-84.

31. Little JP, Safdar A, Bishop D, et al. An acute bout of highintensity interval training increases the nuclear abundance of PGC- $1 \alpha$ and activates mitochondrial biogenesis in human skeletal muscle. Am J Physiol Regul Integr Comp Physiol. 2011;300:R1303-10.

32. Bartlett JD, Hwa-Joo C, Jeong TS, et al. Matched work highintensity interval and continuous running induces similar increases in PGC- $1 \alpha$ mRNA, AMPK, p38 and p53 phosphorylation in human skeletal muscle. J Appl Physiol. 2012;11:1135-43.

33. Rakobowchuk M, Tanguay S, Burgomaster KA, et al. Sprint interval and traditional endurance training induce similar improvements in peripheral arterial stiffness and flow-mediated dilation in healthy humans. Am J Physiol Regul Integr Comp Physiol. 2008;295:R236-42.

34. Cocks M, Shaw CS, Shepherd SO, et al. Sprint interval and endurance training are equally effective in increasing muscle microvascular density and eNOS content in sedentary males. J Physiol. 2013;591:641-56.

35. McKay BR, Paterson DH, Kowalchuk JM. Effect of short-term high-intensity interval training vs. continuous training on $\mathrm{O}_{2}$ uptake kinetics, muscle deoxygenation, and exercise performance. J Appl Physiol. 2009;107:128-38.

36. Bailey SJ, Wilkerson DP, Dimenna FJ, et al. Influence of repeated sprint training on pulmonary $\mathrm{O}_{2}$ uptake and muscle deoxygenation kinetics in humans. $\mathrm{J}$ Appl Physiol. 2009;106:1875-87.

37. Macpherson RE, Hazell TJ, Olver TD, et al. Run sprint interval training improves aerobic performance but not maximal cardiac output. Med Sci Sports Exerc. 2011;43:115-22. 
38. Jacobs RA, Flück D, Bonne TC, et al. Improvements in exercise performance with high-intensity interval training coincide with an increase in skeletal muscle mitochondrial content and function. J Appl Physiol. 2013;115:785-93.

39. Esfandiari S, Sasson Z, Goodman JM. Short-term high-intensity interval and continuous moderate-intensity training improve maximal aerobic power and diastolic filling during exercise. Eur J Appl Physiol. 2014;114:331-43.

40. Kaminsky LA, Arena R, Beckie TMS, et al. The importance of cardiorespiratory fitness in the United States: the need for a national registry: a policy statement from the American Heart Association. Circulation. 2011;127:652-62.

41. Hazell TJ, Macpherson REK, Gravelle BMR, et al. 10 or 30-s sprint interval training bouts enhance both aerobic and anaerobic performance. Eur J Appl Physiol. 2010;110:153-60.

42. Astorino T, Allen R, Roberson D, et al. Effect of high-intensity interval training on cardiovascular function, $\mathrm{VO}_{2} \max$ and muscular force. J Strength Cond Res. 2012;26:138-45.

43. Whyte LJ, Gill JMR, Cathcart AJ. Effect of 2 weeks of sprint interval training on health-related outcomes in sedentary overweight/obese men. Metabolism. 2010;59:1421-8.

44. Gist NH, Fedewa MV, Dishman RK, et al. Sprint interval training effects on aerobic capacity: a systematic review and meta-analysis. Sports Med. 2013;44:269-79.

45. Lee D, Sui X, Artero EG, et al. Long-term effects of changes in cardiorespiratory fitness and body mass index on all-cause and cardiovascular disease mortality in men: the Aerobics Center Longitudinal Study. Circulation. 2013;124:2483-90.

46. Sloth M, Sloth D, Overgaard K, et al. Effects of sprint interval training on $\mathrm{VO}_{2}$ max and aerobic exercise performance: A systematic review and meta-analysis. Scand J Med Sci Sports. 2013;23:341-52.

47. Ma JK, Scribbans TD, Edgett BA, et al. Extremely low-volume, high-intensity interval training improves exercise capacity and increases mitochondrial protein content in human skeletal muscle. J Mol Integr Physiol. 2013;3:202-10.

48. Metcalfe RS, Babraj JA, Fawkner SG, et al. Towards the minimal amount of exercise for improving metabolic health: beneficial effects of reduced-exertion high-intensity interval training. Eur J Appl Physiol. 2011;112:2767-75.

49. Gillen JB, Percival ME, Ludzki A, et al. Interval training in the fed or fasted state improves body composition and muscle oxidative capacity in overweight women. Obesity. 2013;21:2249-55.

50. Currie KD, Dubberley JB, McKelvie RS, et al. Low-volume, high-intensity interval training in patients with CAD. Med Sci Sports Exerc. 2013;45:1436-42.

51. Boyd JC, Simpson CA, Jung ME, et al. Reducing the intensity and volume of interval training diminishes cardiovascular adaptation but not mitochondrial biogenesis in overweight/obese men. PLoS One. 2013;8(7):e68091.

52. Babraj JA, Vollaard NBJ, Keast C, et al. Extremely short duration high intensity interval training substantially improves insulin action in young healthy males. BMC Endocr Disord. 2009;9:3-10.

53. Richards JC, Johnson TK, Kuzma JN, et al. Short-term sprint interval training increases insulin sensitivity in healthy adults but does not affect the thermogenic response to beta-adrenergic stimulation. J Physiol. 2010;588:2961-72.

54. Little JP, Gillen JB, Percival M, et al. Low-volume highintensity interval training reduces hyperglycemia and increases muscle mitochondrial capacity in patients with type 2 diabetes. J Appl Physiol. 2011;111:1554-60.

55. Macpherson REK, Hazell TJ, Olver TD, et al. Run sprint interval training improves aerobic performance but not maximal cardiac output. Med Sci Sports Exerc. 2010;43:115-22.
56. Hazell TJ, Hamilton CD, Olver TD, et al. Running sprint interval training induces fat loss in women. Appl Physiol Nutr Metab. 2014;39:944-50.

57. Trapp E, Heydari M, Freund J, et al. The effects of highintensity intermittent exercise training on fat loss and fasting insulin levels of young women. Int J Obes. 2008;32:684-91.

58. Boutcher SH. High-intensity intermittent exercise and fat loss. J Obes. 2011;2011:1-10.

59. Hazell TJ, Olver TD, Hamilton CD, et al. Two minutes of sprintinterval exercise elicits 24-hr oxygen consumption similar to that of $30 \mathrm{~min}$ of continuous endurance exercise. Int J Sport Nutr Exerc Metab. 2012;22:276-83.

60. Skelly LE, Andrews PA, Gillen JB, et al. High intensity interval exercise induces 24 hour energy expenditure similar to traditional endurance exercise despite reduced time commitment. Appl Physiol Nutr Metab. 2014;39:845-8.

61. Williams CB, Zelt JG, Castellani LN, et al. Changes in mechanisms proposed to mediate fat loss following an acute bout of high-intensity interval and endurance exercise. Appl Physiol Nutr Metab. 2013;38:1236-44.

62. Esbjörnsson-Liljedahl M, Sundberg CJ, Norman B, et al. Metabolic response in type I and type II muscle fibers during a 30-s cycle sprint in men and women. J Appl Physiol. 1999;87:1326-32.

63. Gratas-Delamarche A, Le Cam R, Delamarche P, et al. Lactate and catecholamine responses in male and female sprinters during a Wingate test. Eur J Appl Physiol. 1994;68:362-6.

64. Esbjörnsson-Liljedahl M, Bodin K, Jansson E. Smaller muscle ATP reduction in women than in men by repeated bouts of sprint exercise. J Appl Physiol. 2002;93:1075-83.

65. Jaworowski A, Porter MM, Holmbäck AM, et al. Enzyme activities in the tibialis anterior muscle of young moderately active men and women: relationship with body composition, muscle cross-sectional area and fibre type composition. Acta Physiol Scand. 2002;176:215-25.

66. Esbjörnsson Liljedahl M, Holm I, Sylvén C, et al. Different responses of skeletal muscle following sprint training in men and women. Eur J Appl Physiol Occup Physiol. 1996;74:375-83.

67. Hill DW, Smith JC, Texas N. Gender difference in anaerobic capacity: role of aerobic contribution. $\mathrm{Br} \mathrm{J}$ Sports Med. 1993;27:45-8.

68. Scalzo RL, Peltonen GL, Binns SE, et al. Greater muscle protein synthesis and mitochondrial biogenesis in males compared with females during sprint interval training. FASEB J. 2014;28:1-10.

69. Tarnopolsky MA. Sex differences in exercise metabolism and the role of 17-beta estradiol. Med Sci Sports Exerc. 2008;40:648-54.

70. Hawley JA, Burke LM, Phillips SM, et al. Nutritional modulation of training-induced skeletal muscle adaptations. J Appl Physiol. 2011;110:834-45.

71. Gibala MJ. Nutritional strategies to support adaptation to highintensity interval training in team sports. Nestle Nutr Inst Workshop Ser. 2013;75:41-9.

72. Bishop D. Dietary supplements and team-sport performance. Sports Med. 2010;40:995-1017.

73. Mujika I, Burke LM. Nutrition in team sports. Ann Nutr Metab. 2010;57(Suppl 2):26-35.

74. Stellingwerff T, Maughan RJ, Burke LM. Nutrition for power sports: Middle-distance running, track cycling, rowing, canoeing/kayaking, and swimming. J Sports Sci. 2011;29(Suppl 1):S79-89.

75. Hawley JA, Burke LM. Carbohydrate availability and training adaptation: effects on cell metabolism. Exerc Sport Sci Rev. 2010;38:152-60. 
76. Hansen AK, Fischer CP, Plomgaard P, et al. Skeletal muscle adaptation: Training twice every second day vs. training once daily. J Appl Physiol. 2005;98:93-9.

77. Hulston CJ, Venables MC, Mann $\mathrm{CH}$, et al. Training with low muscle glycogen enhances fat metabolism in well-trained cyclists. Med Sci Sports Exerc. 2010;42:2046-55.

78. Morton JP, Croft L, Bartlett JD, et al. Reduced carbohydrate availability does not modulate training-induced heat shock protein adaptations but does upregulate oxidative enzyme activity in human skeletal muscle. J Appl Physiol. 2009;106:1513-21.

79. Yeo WK, Paton CD, Garnham AP, et al. Skeletal muscle adaptation and performance responses to once a day versus twice every second day endurance training regimens. J Appl Physiol. 2008;105:1462-70.

80. Yeo WK, McGee SL, Carey AL, et al. Acute signaling responses to intense endurance training commenced with low or normal muscle glycogen. Exp Physiol. 2010;95:351-8.

81. Cochran AJ, Little JP, Tarnopolsky MA, et al. Carbohydrate feeding during recovery alters the skeletal muscle metabolic response to repeated sessions of high-intensity interval exercise in humans. J Appl Physiol. 2010;108:628-36.

82. Bartlett JD, Louhelainen J, Iqbal Z, et al. Reduced carbohydrate availability enhances exercise-induced p53 signaling in human skeletal muscle: Implications for mitochondrial biogenesis. Am J Physiol Regul Integr Comp Physiol. 2013;304:R450-8.

83. Polekhina G, Gupta A, Michell BJ, et al. AMPK beta subunit targets metabolic stress sensing to glycogen. Curr Biol. 2003;13:867-71.

84. Polekhina G, Gupta A, van Denderen BJ, et al. Structural basis for glycogen recognition by AMP-activated protein kinase. Structure. 2005;13:1453-62.

85. McBride A, Ghilagaber S, Nikolaev A, et al. The glycogenbinding domain on the AMPK beta subunit allows the kinase to act as a glycogen sensor. Cell Metab. 2009;9:23-34.

86. Bogdanis GC, Nevill ME, Boobis LH, et al. Contribution of phosphocreatine and aerobic metabolism to energy supply during repeated sprint exercise. J Appl Physiol. 1996;80:876-84.

87. Parolin ML, Chesley A, Matsos MP, et al. Regulation of skeletal muscle glycogen phosphorylase and PDH during maximal intermittent exercise. Am J Physiol. 1999;277:E890-900.

88. Spriet LL, Lindinger MI, McKelvie RS, et al. Muscle glycogenolysis and $\mathrm{H}+$ concentration during maximal intermittent cycling. J Appl Physiol. 1989;66:8-13.

89. Mendez-Villanueva A, Edge J, Suriano R, et al. The recovery of repeated-sprint exercise is associated with $\mathrm{PCr}$ resynthesis, while muscle $\mathrm{pH}$ and EMG amplitude remain depressed. PLoS One. 2012;7:e51977.

90. Chin ER, Allen DG. The contribution of pH-dependent mechanisms to fatigue at different intensities in mammalian single muscle fibres. J Physiol. 1998;512:831-40.

91. Fabiato A, Fabiato F. Effects of $\mathrm{pH}$ on the myofilaments and the sarcoplasmic reticulum of skinned cells from cardiace and skeletal muscles. J Physiol. 1978;276:233-55.

92. Bangsbo J, Madsen K, Kiens B, et al. Effect of muscle acidity on muscle metabolism and fatigue during intense exercise in man. J Physiol. 1996;495:587-96.

93. Westerblad H, Bruton JD, Lannergren J. The effect of intracellular $\mathrm{pH}$ on contractile function of intact, single fibres of mouse muscle declines with increasing temperature. J Physiol. 1997;500:193-204.

94. Bishop D, Edge J, Goodman C. Muscle buffer capacity and aerobic fitness are associated with repeated-sprint ability in women. Eur J Appl Physiol. 2004;92:540-7.

95. Weston AR, Myburgh KH, Lindsay FH, et al. Skeletal muscle buffering capacity and endurance performance after high- intensity interval training by well-trained cyclists. Eur J Appl Physiol Occup Physiol. 1997;75:7-13.

96. Hollidge-Horvat MG, Parolin ML, Wong D, et al. Effect of induced metabolic alkalosis on human skeletal muscle metabolism during exercise. Am J Physiol Endocrinol Metab. 2000;278:E316-29.

97. Bishop D, Edge J, Davis C, et al. Induced metabolic alkalosis affects muscle metabolism and repeated-sprint ability. Med Sci Sports Exerc. 2004;36:807-13.

98. Sostaric SM, Skinner SL, Brown MJ, et al. Alkalosis increases muscle $\mathrm{K}^{+}$release, but lowers plasma $\left[\mathrm{K}^{+}\right]$and delays fatigue during dynamic forearm exercise. J Physiol. 2006;570:185-205.

99. Raymer GH, Marsh GD, Kowalchuk JM, et al. Metabolic effects of induced alkalosis during progressive forearm exercise to fatigue. J Appl Physiol. 2004;96:2050-6.

100. Street D, Nielsen JJ, Bangsbo J, et al. Metabolic alkalosis reduces exercise-induced acidosis and potassium accumulation in human skeletal muscle interstitium. $\mathbf{J}$ Physiol. 2005;566:481-9.

101. Carr AJ, Hopkins WG, Gore CJ. Effects of alkalosis and acidosis on performance: a meta-analysis. Sports Med. 2011;41:801-14.

102. Saunders B, Sale C, Harris RC, et al. Sodium bicarbonate and high-intensity-cycling capacity: variability in responses. Int $\mathrm{J}$ Sports Physiol Perform. 2014;9:627-32.

103. Edge J, Bishop D, Goodman C. Effects of chronic NaHCO3 ingestion during interval training on changes to muscle buffer capacity, metabolism, and short-term endurance performance. J Appl Physiol. 2006;101:918-25.

104. Thomas C, Bishop D, Moore-Morris T, et al. Effects of highintensity training on MCT1, MCT4, and NBC expressions in rat skeletal muscles: Influence of chronic metabolic alkalosis. Am J Physiol Endocrinol Metab. 2007;293:E916-22.

105. Bishop DJ, Thomas C, Moore-Morris T, et al. Sodium bicarbonate ingestion prior to training improves mitochondrial adaptations in rats. Am $\mathbf{J}$ Physiol Endocrinol Metab. 2010;299:E225-33.

106. Sutton JR, Jones NL, Toews CJ. Effect of $\mathrm{pH}$ on muscle glycolysis during exercise. Clin Sci. 1981;61:331-8.

107. Stephens TJ, McKenna MJ, Canny BJ, et al. Effect of sodium bicarbonate on muscle metabolism during intense endurance cycling. Med Sci Sports Exerc. 2002;34:614-21.

108. Bouissou P, Defer G, Guezennec CY, et al. Metabolic and blood catecholamine responses to exercise during alkalosis. Med Sci Sports Exerc. 1988;20:228-32.

109. Bezaire V, Heigenhauser GJ, Spriet LL. Regulation of CPT I activity in intermyofibrillar and subsarcolemmal mitochondria from human and rat skeletal muscle. Am J Physiol Endocrinol Metab. 2004;286:E85-91.

110. Starritt EC, Howlett RA, Heigenhauser GJ, et al. Sensitivity of CPT I to malonyl-CoA in trained and untrained human skeletal muscle. Am J Physiol Endocrinol Metab. 2000;278:462-8.

111. van Loon LJ, Greenhaff PL, Constantin-Teodosiu D, et al. The effects of increasing exercise intensity on muscle fuel utilisation in humans. J Physiol. 2001;536:295-304.

112. Jeppesen J, Kiens B. Regulation and limitations to fatty acid oxidation during exercise. J Physiol. 2012;590:1059-68.

113. Hill CA, Harris RC, Kim HJ, et al. Influence of beta-alanine supplementation on skeletal muscle carnosine concentrations and high intensity cycling capacity. Amino Acids. 2007;32:225-33.

114. Hobson RM, Saunders B, Ball G, et al. Effects of beta-alanine supplementation on exercise performance: a meta-analysis. Amino Acids. 2012;43:25-37.

115. Gross M, Boesch C, Bolliger CS, et al. Effects of beta-alanine supplementation and interval training on physiological determinants of severe exercise performance. Eur J Appl Physiol. 2014;114:221-34. 
116. Smith AE, Walter AA, Graef JL, et al. Effects of beta-alanine supplementation and high-intensity interval training on endurance performance and body composition in men; a double-blind trial. J Int Soc Sports Nutr. 2009;6:18.

117. Walter AA, Smith AE, Kendall KL, et al. Six weeks of highintensity interval training with and without beta-alanine supplementation for improving cardiovascular fitness in women. J Strength Cond Res. 2010;24:1199-207.

118. Trost SG, Owen N, Bauman AE, et al. Correlates of adults' participation in physical activity: review and update. Med Sci Sports Exerc. 2002;34:1996-2001.
119. Bartlett JD, Close GL, MacLaren DP, et al. High-intensity interval running is perceived to be more enjoyable than moderate-intensity continuous exercise: implications for exercise adherence. J Sports Sci. 2011;29:547-53.

120. van Loon LJ, Tipton KD. Concluding remarks: nutritional strategies to support the adaptive response to prolonged exercise training. Nestle Nutr Inst Workshop Ser. 2013;75:135-41. 